\title{
Importancia de los índices simplificados en el diagnóstico y estudio de los Trastornos Temporomandibulares
}

\author{
Importance of the rates simplified in the diagnosis and study of the temporomandibular disorders
}

\author{
Sergio Alvarado-Menacho ${ }^{1, a}$
}

\section{RESUMEN}

Desde que se definieron a los Trastornos Temporomandibulares (TTMs), en la época de Costen, ha sido muy difícil poder identificarlos y diferenciarlos, en un primer momento se les estudió desde el punto de vista clínico pero al observar su origen multifactorial y ver que era un problema que afectaba a gran parte de la población, se le dio una mayor importancia desde el punto de vista epidemiológico; se clasificaron y se estudiaron enfocando dos grandes áreas, una los de origen muscular y la otra de origen óseo articular, las herramientas de estudio clínico se volvieron cada vez más complejas y posteriormente al observar que tenían un componente psicosocial, se extendió el estudio hacia ese campo. El objetivo de ésta revisión es mostrar la importancia que tienen los índices y test simplificados en el diagnóstico y estudio epidemiológico de los Trastornos Temporomandibulares. Se realizó una búsqueda No sistemática de trabajos relacionados con el tema de índices y test simplificados y validados en el estudio de los TTMs. Existen distintos índices con los cuales se pueden identificar los TTMs y que permiten poder realizar estudios epidemiológicos a gran escala los que habiendo sido validados con exámenes clínicos más detallados permiten obtener resultados similares. Los índices simplificados especialmente el índice simplificado de Fonseca permiten identificar y estudiar tanto clínica como epidemiológicamente los TTMs.

PALABRAS CLAVE: Articulación temporomandibular, trastornos de la articulación temporomandibular, síndrome de la disfunción de articulación temporomandibular.

\section{SUMMARY}

Since the temporomandibular disorders (TTMs), was identified at the time of Costen, it has been very difficult to be able to identify and differentiate them, at first we studied them from the clinical point of view but to observe its multifactorial origins and see that it was a problem affecting a large part of the population, it was given a more 
importance consideration from an epidemiological point of view; they were classified and they were focused on two main areas, a muscular origin and an articular origen, clinical study tools became increasingly complex and then watching that there is a psychosocial component, is was extended the study to that field. The objective of this review is to show the importance of indexes and test simplified on the diagnosis and epidemiological study of the temporomandibular disorders. A search was conducted No systematic work related to the theme of indexes and test simplified and validated in the study of the TTMs. There are various indices with which the TTMs can be identified and which allow to carry out studies epidemiological large-scale which having been validated with clinical tests more detailed you get similar results. Simplified indexes especially the simplified index of Fonseca allow identify and study both clinical and epidemiologically the TTMs.

\section{KEY WORDS: Temporomandibular joint, temporomandibular joint disorders, temporomandibular joint dysfunction syndrome.}

\section{INTRODUCCIÓN}

Es bien reconocido que dentro de los problemas más prevalentes de salud oral se encuentran: la caries dental, la enfermedad periodontal y las maloclusiones, y ello no escapa de nuestra población en Perú, pero no podemos dejar de lado que los Trastornos Temporomandibulares (TTMs), en orden de importancia son las alteraciones que le siguen en orden de prevalencia siendo la causa más importante de dolor que no es de origen dentario dentro del sistema estomatognático.

Desde la primera vez que se reportaron problemas auditivos asociados a perdida de piezas dentarias en el año 1934 como lo hizo el Dr. James Costen(1) en la que asoció estos dos aspectos en 11 pacientes, hasta la fecha ha habido muchos intentos de identificar los problemas de los TTMs. Para el diagnóstico de los TTMs de hace mucho tiempo se han confeccionado diversos índices para su diagnóstico, siendo el más usado y de mayor aceptación el Índice de Helkimo(2), que fue modificado por Maglione(3) en 1986 con la finalidad de obtener mejores resultados en su distribución de severidad.

Podemos encontrar en la literatura como se hacen muchos intentos de estudiar los TTMs mediante instrumentos de diagnóstico que en su mayoría eran para ser aplicados en la clínica(3-11)prospectivo, transversal y descriptivo en 37 ancianos de 60 a 80 años para conocer los signos y síntomas de los trastornos temporomandibulares que se presentan con mayor frecuencia en este grupo etario. Los signos y síntomas que se valoraron fueron: dolor, ruidos articulares, bloqueos, limitación funcional de la apertura mandibular, desgaste dental, pérdida de dientes, dimensión vertical, colapso posterior de la mordida y rehabilitación protésica, entre otros. El 46\% ( $\mathrm{n}=17$ y algunos en estudios epidemiológicos(12,13) a gran escala pero pocos eran de tipo anamnésico, dentro de estos últimos podemos rescatar el Índice Anamnésico de Fonseca, el que fue confeccionado y validado por el Dr. Dickson da Fonseca en San Pablo en 1992(14,15) y replicada su validación por Lázaro(16,17) en Lima el 2009.

Los índices anamnésicos, tienen la ventaja que pueden ser utilizados en poblaciones mayores y su aplicación se realiza en menor cantidad de tiempo $(16,18)$; esta situación nos permite realizar una adecuada evaluación epidemiológica para poder identificar casos reales de TTMs dentro de la población y no perder el tiempo en el estudio de falsos positivos, para permitir un examen clínico a mayor profundidad en los verdaderos positivos, y de paso nos sirve para su utilización en la práctica clínica diaria. También se han estado haciendo estudios en base a dos ejes distintos como son el eje físico (Eje I) y el eje psicosocial (Eje II)(19-21), dicho sea de paso para éstos estudios se utiliza un cuestionario anamnésico en el eje I.

El objetivo de esta revisión fue demostrar la relevancia que tienen los índices simplificados en el estudio de los Trastorno Temporomandibulares facilitando la labor tanto del clínico como del epidemiólogo en el diagnóstico de los mismos.

Uno de los primeros en proponer un test que mediante ciertos parámetros trataba de estudiar a través de un breve examen clínico y preguntas anamnésicas fue Krogh-Poulsen(22), estableciendo un formato de nueve ítems que definían mediante una evaluación cuanti-cualitativa ciertos rangos que identificaban 
si el paciente era un paciente disfuncionado o no lo era, ya que para obtener un verdadero positivo tenía que tener tres aspectos de éste test como positivos; hay que tener en cuenta que en esa época (1968), existía mucha controversia con respecto a la posición articular de la relación céntrica y por ésta razón dicha posición articular no estaba claramente definida y el Dr.Krogh-Poulsen(23), indicaba su ítem $\mathrm{N}^{\circ}$ 7(el que se refería al primer contacto en céntrica), como un punto positivo en los aspectos disfuncionales, aspecto clínico que en la actualidad solamente se considera como positivo si es que excede los valores permitidos de normalidad.

Desde ya realizar la medición de los parámetros clínicos, exigía una calibración adecuada con la finalidad de poder valorar adecuadamente los problemas y mediciones encontrados. Helkimo(2), utilizo los mismos parámetros que el test de Krogh Poulsen(23), pero fue un poco más allá en el sentido que catalogó el grado de severidad de ellos realizando un índice (Índice de Disfunción Clínica Craneomandibular IDCCM- o índice de Helkimo(10) que utilizaba 5 ítems los cuales se categorizaban como 0 al que no tenía inconvenientes, 1 al que tenía alteraciones leves y 5 al que presentaba grandes alteraciones, haciendo de éste índice que era netamente clínico a pesar que utilizaba algunas preguntas de tipo cualitativas, una herramienta más exacta en el diagnóstico de los problemas disfuncionales ya que valoraba en forma cuantitativa los distintos puntos de análisis.

Muchos trabajos utilizaron el Índice de Helkimo como herramienta de diagnóstico como fue Arroyo que estudió la influencia de las desarmonías oclusales sobre la sintomatología de los TTMs en estudiantes de Odontología de la Universidad Nacional Mayor de San Marcos (9). El autor seleccionó al azar 205 estudiantes de ambos sexos, y se halló una prevalencia de $46.8 \%$ de TTMs. Si éste estudio se hubiera querido aplicar a nivel de una población mayor hubiera requerido una inversión mucho mayor de tiempo y dinero además de personal capacitado para poder ejecutar el índice de Helkimo, situación que lo hubiera convertido en inviable, y esta es la razón por la cual los resultados de este tipo de trabajo no se pueden extrapolar a una población mayor.

\section{DISCUSIÓN}

Olga Taboada el 2002, realizó un estudio epidemiológico en 37 adultos mayores de 60 a 80 años de edad, independientes y relativamente sanos (con una enfermedad crónica menor p. ej. hipertensión arterial), pertenecientes a una clase social media y medio baja, residentes del municipio de los Reyes la Paz en el Estado de México y que participan en los núcleos gerontológicos de la Unidad de Investigación en Gerontología de la Facultad de Estudios Superiores Zaragoza, en el que aplicó solamente examen clínico, concluyendo que los TTM pueden ser considerados como una alteración frecuente en la población senecta y con una alta necesidad de tratamiento (4).

Javier Rubio relacionó el buceo como factor de riesgo en la prevalencia de trastornos temporomandibulares musculares y articulares, estudio a 39 miembros del personal militar que labora en la Marina de Guerra del Perú como buzos profesionales, utilizó para su estudio el índice de Helkimo, encontrando que el $91.42 \%$ de buzos objeto del estudio presentaron TTMs, musculares y articulares(6), podemos apreciar que la muestra fue pequeña ya que al aplicar el índice de Helkimo obligaba a un exhaustivo examen clínico.

Claudia Hormiga et al., realizaron un estudio sobre prevalencia de síntomas y signos de trastornos temporomandibulares en una población universitaria del área metropolitana de Bucaramanga en Santander donde estudio a 260 estudiantes universitarios y aplicando el índice de Helkimo, concluyendo que con los datos recolectados fue posible proporcionar un panorama de la frecuencia y algunos factores relacionados a los TTM (5); como vemos, la muestra se mantiene dentro de la cantidad de la mayoría de los estudios epidemiológicos.

Medina el año 2010 realizó un estudio sobre prevalencia de trastornos temporomandibulares y su relación con la pérdida de soporte oclusal posterior en adulto(7), utilizando el índice de Helkimo en 400 pacientes mayores de 25 años que acudieron al servicio de Odontoestomatología del Centro Médico Naval en los meses de Febrero, Marzo y Abril del año 2010, concluyendo que el $83 \%$ de los pacientes con pérdida de soporte oclusal posterior, y el $73 \%$ de los pacientes sin pérdida de soporte oclusal posterior presentaron trastornos temporomandibulares. Podemos apreciar 
que Medina utilizó una muestra mayo (400 pacientes), pero a pesar de ello es una muestra pequeña que no nos permite transpolar los resultados a poblaciones mayores.

Fabiola Ortiz el 2012(3), realizó un estudio sobre factores asociados a la disfunción temporomandibular en la clínica de tres Facultades de odontología en la Universidad Veracruzana campus Minatitlám en Méjico, con una asistencia de 348 pacientes utilizando el índice de Helkimo pero modificado por Maglione, concluyendo que un $31 \%$ de los pacientes estudiados presentaron TTMs. Al igual que los trabajos anteriores la muestra fue pequeña lo que nos imposibilita poder comparar estos resultados o traspolarlos con poblaciones mayores.

Thais Chaves et al., nos mencionan que en la literatura especializada existen variados instrumentos para la evaluación de la disfunción temporomandibular (DTM) como índices, cuestionarios, protocolos, escalas de evaluación y criterios de diagnóstico, en su revisión presentaron dos índices y tres cuestionarios (anamnésicos y funcionales) (10). También nos indica que los índices son herramientas que organizan la evaluación de signos y síntomas, a través de la obtención de puntuaciones, en cambio los cuestionarios son mejor aplicados para trazar perfiles poblacionales en estudios epidemiológicos. Aunque menciona que, para la evaluación de los eventuales impactos de los DTM en las actividades de la vida diaria, los cuestionarios funcionales son los más adecuados. Dentro de los índices que nos presenta Thais Chaves menciona: el Índice de Disfunción Clínica Craneomandibular - IDCCM- o índice de Helkimo(2), Índice Craneomandibular (ICM) o Índice Temporomandibular (ITM) propuesto por Fricton y Schiffman (24), el Cuestionario e Índice Anamnésico de Fonseca $(14,15)$, Cuestionario de la Academia Americana de Dolor Orofascial (25), el Cuestionario e Índice de Limitación Funcional Mandibular (MFIQ). Concluyendo que los cuestionarios son mejor empleados en estudios epidemiológicos, para trazar perfiles poblacionales, para hacer triage inicial de pacientes, o para la evaluación de calidad de vida.

En nuestro país, se han realizado varios estudios desarrollando los índices (o cuestionarios) anamnésicos simplificados como fue el trabajo de Lázaro el 2009(17), en el cual se validó el índice anamnésico simplificado de Fonseca para el diagnóstico de trastornos temporomandibulares en la que se trabajó con una muestra de 200 pacientes mayores de 18 años que acudieron al Servicio de Odontoestomatología del Hospital Nacional "Luís N. Sáenz" de la Policía Nacional del Perú durante los meses de julio y agosto del 2008, la validación se realizó con el índice de Helkimo como gold estándar, concluyendo que tiene una sensibilidad del 96\%, una especificidad de 95\%, un valor predictivo positivo del $97 \%$ y encontrado mayor frecuencia de TTM entre las mujeres con el $66,7 \%$ frente el $43,7 \%$ entre los hombres, como vemos la muestra fue relativamente pequeña ya que era un estudio de validación y tenía que compararse con un índice clínico.

Erick Chacaltana también utilizó el índice anamnésico simplificado de Fonseca en el 2015 realizando un estudio sobre prevalencia de trastornos temporomandibulares según el índice anamnésico simplificado de Fonseca en pacientes con diagnóstico de artritis reumatoide del hospital nacional dos de mayo(18), en el que trabajó con una muestra de pacientes de 20 a 90 años diagnosticados con artritis reumatoide que asistieron a los consultorios externos del servicio de Reumatología e Inmunología del Hospital Nacional Dos de Mayo y que de acuerdo a los registros estadísticos del HNDM del periodo 2009-2013, en promedio fueron 250 pacientes evaluados al mes. Concluyendo que existe presencia de TTM en el $85.3 \%$ de los pacientes evaluados y de ellos el $49 \%$ presentó TTM Leve, $27.5 \%$ TTM moderado y el $8.8 \%$ TTM severo.

Más recientemente, se han realizado trabajos como el de Cárol Diaz en el año $2017^{(20)}$, en el que utilizó los criterios de diagnóstico DC/TMD, éstos estudios a pesar que pueden ser aplicados poblacionalmente, requieren de una preparación y calibración mayor por parte del examinador clínico ya que trabaja en base a dos ejes, el Eje $\mathrm{N}^{\circ}$ I que ve los aspectos físicos (examen clínico) y el Eje $\mathrm{N}^{\circ}$ II que ve los aspectos psicosociales, siendo más difícil su aplicación a gran escala aunque su sensibilidad y especificidad es bastante alta.

El autor no posee conflictos de interés en ésta publicación. 


\section{CONCLUSIONES}

Podemos apreciar que las conclusiones de los resultados de la prevalencia de los Trastornos temporomandibulares en los distintos trabajos realizados en nuestro país como en el extranjero, son bastante similares manteniéndose entre los rangos de prevalencia a nivel mundial, los estudios dicho sea de paso han sido realizados mediante índices clínicos, índices anamnésicos o cuestionarios.

Los índices o cuestionarios anamnésicos al haber sido validados con los índices clínicos nos pueden dar resultados que tienen validez en la aplicación de estudios epidemiológicos en grandes poblaciones, sin el inconveniente del uso de personal altamente capacitado ni el desembolso de gran cantidad de esfuerzo y dinero en su aplicación, obteniendo resultados que si pueden ser aplicados a nivel poblacional ya que permiten el acceso a mayor cantidad de personas sin el perjuicio de perder sensibilidad y especificidad. Por ello son de gran utilidad tanto clínica, como epidemiológica, así como para el uso de triaje y evaluación de calidad de vida.

Por todo lo expuesto recomiendo el uso de los índices anamnésicos simplificados en especial en estudios epidemiológicos de los Trastornos Temporomandibulares aplicables a grandes poblaciones.

\section{Correspondencia:}

Sergio Alvarado Menacho

Correo electrónico: salvaradom@unmsm.edu.pe

Agradecimientos: el autor agradece el apoyo prestado en la realización de esta revisión a las Dras. Sandra Palomino Gómez, Laura Ramírez Sotelo y Karin Uchima Koecklin, docentes del Doctorado en Estomatología de la UNMSM.

\section{REFERENCIAS BIBLIOGRÁFICAS}

1. Costen JB. Syndrome of ear and sinus symptoms dependent upon disturbed functions of the temporomandibular joint. 1934. Ann Otol Rhinol Laryngol. 1997;106(10 Pt 1):805-819. DOI: 10.1177/000348949710601002

2. Helkimo M. Studies on function and dysfunction of the masticatory system. IV. Age and sex distribution of symptoms of dysfunction of the masticatory system in Lapps in the north of Finland. Acta Odontol Scand. 1974;32(4):255-67.

3. Ortiz F, Martínez C, Ríos M, Alvarado M, Pérez G. Factores asociados a la disfunción temporomandibular. Odontol actual. 2012;9(111):16-22.

4. Taboada O, Gomez Y, Taboada S, Mendoza V. Prevalencia de signos y sintomas de los trastornos temporomandibulares en un grupo de adultos mayores. Rev la Asoc Dent Mex. 2004;61(4):125-9. (Fecha de acceso 15 de marzo del 2019)Disponible en: http://www. medigraphic.com/pdfs/adm/od-2004/od044b.pdf

5. Hormiga C, Bonet M, Alodia C, Jaimes A. Prevalencia de síntomas y signos de trastornos temporomandibulares en una población universitaria del área metropolitana de Bucaramanga, Santander. Umbral Científico. 2009;14:80-91. (Fecha de acceso 15 de marzo del 2019)Disponible en: http://www.redalyc.org/articulo. oa?id=30415059007\%

6. Rubio J. El buceo como factor de riesgo en la prevalencia de trastornos temporomandibulares musculares y articulares. Tesis. Lima, Perú: Universidad Nacional Mayor de San Marcos; 2007.

7. Medina A. Prevalencia de trastornos temporomandibulares y su relacion con la pérdida de soporte oclusal posterior en adultos.- Tesis. Lima, Perú: Universidad Nacional Mayor de San Marcos; 2010. (Fecha de acceso 15 de marzo del 2019)Disponible en: http://cybertesis.unmsm.edu.pe/bitstream/cybertesis/2199/1/ Medina_sa.pdf

8. Paredes G. Distribución de signos y síntomas de la disfunción del sistema estomatognático y su relación con algunos factores predisponentes. Tesis. Lima, Perú: Universidad Nacional Mayor de San Marcos; 1988.

9. Arroyo C. Relación entre signos y síntomas de Desordenes Temporomandibulares y disarmonías oclusales en estudiantes de Odontología de la Universidad Nacional Mayor de San Marcos. Tesis. Lima, Perú: Universidad Nacional Mayor de San Marcos; 1999.

10. Chaves T, De Oliveira A, Grossi D. Principais instrumentos para avaliação da disfunção temporomandibular, parte I : índices e questionários; uma contribuição para a prática clínica e de pesquisa. Fisioter e Pesqui. 2008;15(1):92-100.

11. Acosta R. Una revisión de la literatura sobre la relación causal entre los factores oclusales (FO) y los desórdenes temporomandibulares (DTM) V: efecto de los cambios en los factores oclusales conseguidos con el tratamiento de ortodoncia. Rev Fac Odontol Univ Antioq. 2011;22:205-26.

12. Okeson J. Etiología de los trastornos funcionales del sistema masticatorio. La Carta Odontológica. 1995; 11: $22-8$

13. Okeson J. Tratamiento de oclusión y afecciones temporomandibulares. 7ma. ed. Barcelona: Elsevier; 2013. p.20. 
14. Fonsêca D. Disfunçäo craniomandibular - (DCM): diagnóstico pela anamnese. Tesis de Maestria. Sao Paulo: Universidade de Säo Paulo; 1992.

15. Fonsêca D, Bonfante G, Valle A, de Freitas S. Diagnóstico pela anamnese da disfunção craniomandibular. Rev Gauch Odontol. 1994;4(1):23-32.

16. Lazaro J. Validación del índice anamnésico simplificado de Fonseca para el diagnóstico de trastornos temporomandibulares. Tesis. Lima, Perú: Universidad Nacional Mayor de San Marcos; 2008.

17. Lazaro-Valdiviezo J, Alvarado-Menacho S. Validación del índice anamnésico simplificado de Fonseca para el diagnóstico de trastornos temporomandibulares. Odontol Clín-Cientif, Recife. 2009;8(2):163-8.

18. Chacaltana E. Prevalencia de trastornos temporomandibulares según el índice reumatoide del Hospital Nacional Dos De Mayo. Tesis. Lima, Perú: Universidad Nacional Mayor de San Marcos; 2015.

19. Stumpf R, Stumpf C, De Sousa R, Rapoport A. Freqüência de relatos de parafunções nos sub- grupos diagnósticos de DTM de acordo com os critérios diagnósticos para pesquisa em disfunções temporomandibulares (RDC/TMD). Rev Dent Press Ortod e Ortop Facial. 2008;13(2):61-9. Doi: http://dx.doi. org/10.1590/S1415-54192008000200008

20. Diaz C. Características oclusales y disfunción temporomandibular según los criterios de diagnóstico dc/ tmd en pacientes adultos jóvenes Tesis. Lima, Perú: Universidad Nacional Mayor de San Marcos; 2017. (Fecha de acceso 15 de marzo del 2019)Disponible en: http://200.62.146.130/handle/cybertesis/6419
21. Barakat K. Correlation between pain and arthroscopic features of synovitis: A logical approach to verify correlations of TMJ pain. Tanta Dent J. 2013;10(3):16872. Doi: https://doi.org/10.1016/j.tdj.2013.12.005

22. Sardiña M, Casa J, Martínez I, Peñate C, Peñate D. Factores de riesgo de la disfunción temporomandibular asociados al Test de Krogh Paulsen. Rev Méd Electrón [Internet]. 2010;32(5):0-0. (Fecha de acceso 15 de marzo del 2019)Disponible en: http://www. revmatanzas.sld.cu/revista medica/ano 2010/vol5 2010/tema04.htm

23. Schwartz L, Chayes C. Facial pain and mandibular dysfunction. Philadelphia: W.B. Saunders Co.; 1968. p. 236-280.

24. Schiffman EL, Fricton JR, Haley DP, Shapiro BL. The prevalence and treatment needs of subjects with temporomandibular disorders. J Am Dent Assoc. 1990;120(3):295-303. Doi: https://doi.org/10.14219/ jada.archive. 1990.0059

25. Reiter S, Goldsmith C, Emodi-Perlman A, Friedman-Rubin P, Winocur E. Masticatory muscle disorders diagnostic criteria: The American Academy of Orofacial Pain versus the research diagnostic criteria/ temporomandibular disorders (RDC/TMD). J Oral Rehabil. 2012;39(12):941-7. Doi: doi: 10.1111/j.13652842.2012.02337.x

Recibido: 19-07-2018

Aceptado: 03-11-2018 